\title{
Tunepal: Searching a Digital Library of Traditional Music Scores
}

\author{
Bryan Duggan \\ Technological University Dublin, bryan.duggan@tudublin.ie \\ Brendan O'Shea \\ Technological University Dublin, brendan.oshea@tudublin.ie
}

Follow this and additional works at: https://arrow.tudublin.ie/scschcomrep

Part of the Celtic Studies Commons, Ethnomusicology Commons, Musicology Commons, and the Music Practice Commons

\section{Recommended Citation}

Bryan Duggan, Brendan O'Shea, (2011) "Tunepal: searching a digital library of traditional music scores", OCLC Systems \& Services, Vol. 27 Iss: 4, pp.284 - 297

This Other is brought to you for free and open access by the School of Computer Sciences at ARROW@TU Dublin. It has been accepted for inclusion in Reports by an authorized administrator of ARROW@TU Dublin. For more information, please contact arrow.admin@tudublin.ie, aisling.coyne@tudublin.ie, gerard.connolly@tudublin.ie.

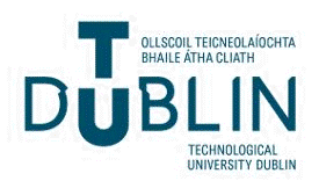


OCLC

27,4

284

Received February 2011

Revised April 2011

Accepted May 2011

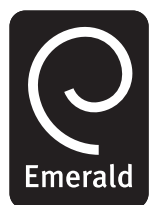

OCLC Systems \& Services: International digital library perspectives

Vol. 27 No. 4, 2011

pp. $284-297$

(C) Emerald Group Publishing Limited 1065-075X

DOI $10.1108 / 10650751111182597$

\section{Tunepal: searching a digital library of traditional music scores}

\author{
Bryan Duggan and Brendan O'Shea
}

School of Computing, Dublin Institute of Technology, Dublin, Ireland

\begin{abstract}
Purpose - This paper aims to describe the Tunepal project as an example of a music information retrieval (MIR) system that is having an impact on how musicians access, learn and play traditional Irish music around the world.

Design/methodology/approach - This paper describes the functionality of the Tunepal system: consisting of the tune corpus, the web site tunepal.org and mobile apps supporting iOS and Android OS. Tunepal facilitates query-by-title and query-by-playing music (QBP) searches and allows a musician to retrieve and playback scores amongst other supported functions.

Findings - Tunepal has been favorably received and musicians report that the system is being used in a variety of scenarios including archiving and the preparation of sleeve notes for commercial recordings. Tunepal has a growing user base in 25 countries.

Originality/value - The comprehensive tune corpus (over 16,000 compositions), the query-by-playing technology and the fact that the mobile apps provide access to the corpus in situ in traditional music sessions and classes make this project uniquely useful.
\end{abstract}

Keywords Music information retrieval, Query-by-playing, Traditional music, Music, Searching, Information retrieval

Paper type Case study

\section{Introduction}

This paper describes Tunepal, a popular search engine and music retrieval tool for musicians playing traditional Irish dance music. Tunepal is accessible free of charge through the web site tunepal.org and through mobile apps available at minimal cost on iOS and Android OS. Tunepal connects musicians to the scores and metadata of 16,700 traditional Irish, Welsh, Scottish, Breton, American marching band and Canadian dance tunes drawn from community sources, such as the web site thesession.org and "standard" references including Aird's Airs, O’Neills Dance Music of Ireland and Breandán Breathnach's Ceol Rince Na hÉireann series. Tunes can be retrieved by typing in a title or through a unique query-by-playing interface that allows users to find tunes by playing a 12 second extract from the tune on a traditional instrument. Tunepal on mobile devices can be used in situ in traditional music sessions, classes and concerts. Tunepal has proven very popular both in Ireland and internationally, with users in 29 countries.

Section 2 of this paper presents some background information on the types of music Tunepal is designed to work with. Section 3 describes the ABC music notation language - the native notation format that Tunepal can import. Section 4 presents the

The Tunepal project is supported by a grant from the Irish Government Department of Tourism, Culture and Sport. Tunepal was developed from research sponsored by the School of Computing, Dublin Institute of Technology. 
features of the tunepal.org web site while section 5 presents the Tunepal mobile apps. Section 6 shows how Tunepal is being used around the world, while section 7 presents conclusions and future work.

\section{Background}

In common with the folk music of many cultures, repertoire in Irish traditional music is primarily acquired aurally. Musicians playing Irish music learn by hearing tunes played by fellow musicians (Wallis and Wilson, 2001) rather than from tune books. Since the 1960's it is common for musicians to play and learn tunes in sessions semi-formal gatherings of musicians and occasionally dancers that often take place in pubs (Figure 1).

Often sessions are anchored by one or two core musicians who may be paid to play, though sessions are generally open to guests of appropriate standard. Because of the significant size of the canon of traditional music (over seven thousand compositions) many musicians playing music in sessions are unaware of the title or origin of the tunes they play (Driscoll, 2004). The most common forms of dance tunes are: reels, double jigs and hornpipes. Other tune types include marches, set dances, polkas, mazurkas, slip jigs, single jigs and reels, flings, highlands, scottisches, barn dances, strathspeys and waltzes (Larsen, 2003). These forms differ in time signature, tempo and structure. For example a reel is generally played at a lively tempo and is in $4 / 4$ time (written as eight quavers in a bar) while a waltz is generally played at slower pace and is in 3/4 time. The time signature, tempo and structure of a tune form are determined by the dance it accompanies. Most tunes consist of a common structure of two parts traditional musicians refer to as the $A$ part and $B$ part. Musicians playing traditional Irish music have a personal repertoire of up to a thousand tunes. Many of these tunes are known by multiple names, while many more are known simply as "gan anim" (without name) (Driscoll, 2004). In the modern age, Irish traditional musicians play fiddles, uilleann pipes, tin-whistles, flutes, concertina's accordions, banjos and harps. Other less popular instruments include the mandolin, harmonica and dulcimer (Vallely, 1999).

\section{Music collections and the $\mathrm{ABC}$ music notation language}

There have been notable initiatives to catalogue the canon of Irish traditional music (Petrie, 1855; Bunting, 1840; Joyce, 1909; Shields, 1998) but the majority of traditional
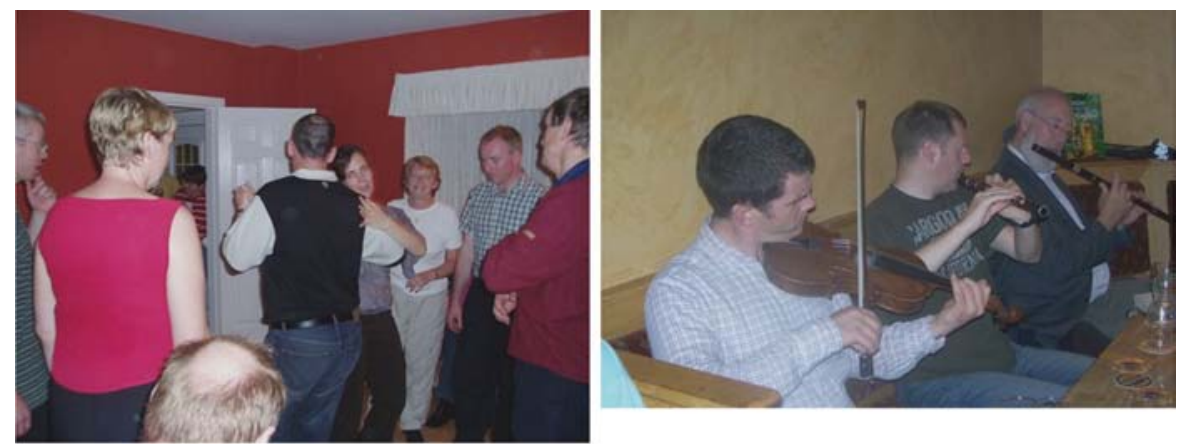

Notes: Set dancers (left). Fiddle player Colm Logue, the author and flute player Patsy Hanley at an informal session at Fleadh Ceol na h'Eireann 2008 (right)

\section{Tunepal: searching a digital library}

285 
OCLC 27,4

286

tunes were not transcribed until the turn of the twentieth century when Francis O'Neill, the then police chief in Chicago, transcribed and documented a large body of dance tunes and airs from immigrant Irish musicians.

In 1903, he published a book of his collected tunes entitled The Music of Ireland. The 1,850 tunes presented in the collection were classified according to tune-type (airs and songs, O'Carolan compositions, double jigs, slip jigs, reels, hornpipes, long dances, marches and miscellaneous). In 1907, he published The Dance Music of Ireland - 1001 Gems. This collection focused entirely on the dance music repertoire and contained many tunes published in his previous collection (Figure 2). O'Neill's second book was considered the definitive source for traditional musicians and musicians would often refer to a tune by its reference number in the book (Wallis and Wilson, 2001).

Breandán Breathnach's Ceol Rince Na hÉreann series in five volumes is regarded as the most significant and influential collection of traditional Irish music after O'Neill's books (Breathnach, 1999). Breathnach's books contain tunes from many sources including field recordings, commercial recordings and manuscript collections of dance music held in private hands.

By identifying duplicates and variations Breathnach sought to identify the earliest occurrences of tunes and trace their history through printed manuscript collections and recordings. His books contain detailed bibliographic notes on each of the tunes included.

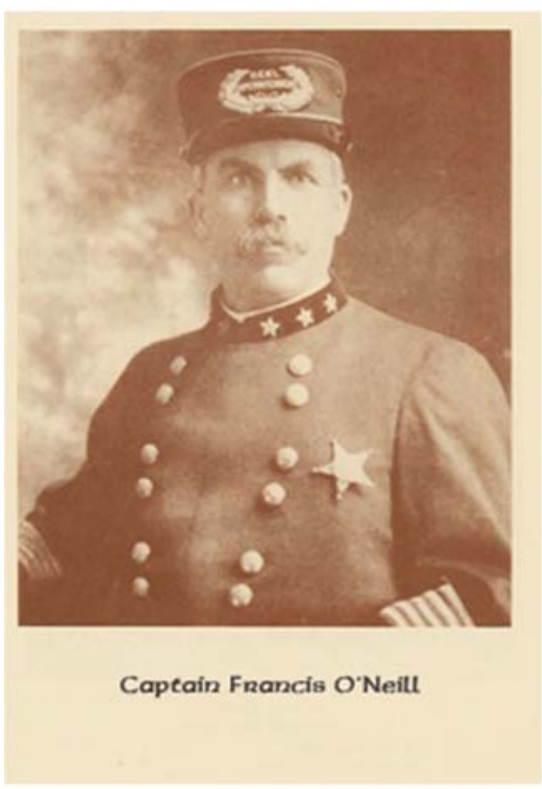

(a)

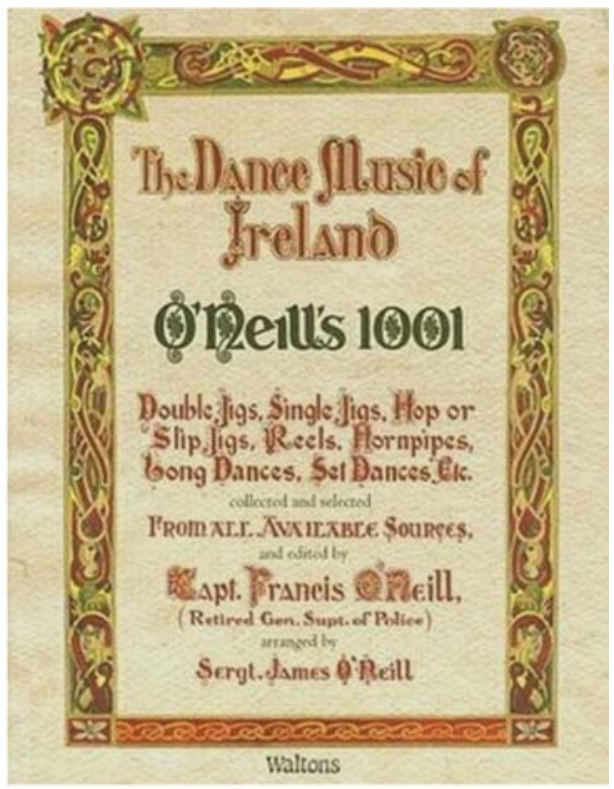

(b)
Police Chief Francis
O'Neill and the cover of O'Neill's The Dance Music of Ireland
Sources: (a) Francis O'Neill, collector/unidentified photographer, Chicago: Lyon and Healy (1903) Copyright: Francis O'Neill (1903). (b) The Dance Music of Ireland, O'Neill, Francis; O’Neill, James, Chicago: Lyon and Healy (1907) 


\section{Collections in electronic format}

$\mathrm{ABC}$ is a music notation language introduced by Chris Walshaw in 1991 (Walshaw, 2011) for typesetting traditional tunes. The format was designed primarily for folk and traditional tunes of Western European origin, which can be written on one stave in standard classical notation (Walshaw, 2011). The tune given in Figure 3 is typical of the transcriptions that can be sourced in $\mathrm{ABC}$ from publicly available databases.

In this transcription the transcriber has helpfully included useful metadata with the notation for the tune such as similar tunes and variations. ABC files are ASCII text files and so can be edited by any text editor, without the necessity for special software. Each file (known as a tunebook) can contain multiple tunes. File sizes are typically measured in kilobytes and this facilitates easy transmission over the internet.

The header section contains amongst other fields, the title, composer, source, tempo, key, geographical origin and transcriber (Mansfield, 2011). As tunes can have several titles, the title field can be repeated for a given tune. The tune body contains the notation for the tune. The body encoding supports such features as ornaments, bar divisions, sharps, flats, naturals, repeated sections, key changes, guitar chords, lyrics and variations. There is an active and vibrant community supporting $\mathrm{ABC}$ notation and a range of tools have been developed for a variety of platforms and purposes.

Between 1997 and 2000, a group of musicians under the leadership of Dan Beimborn and John Chambers, undertook a grass roots project to transcribe three of O'Neill's books to electronic format using ABC notation. As copyright had expired on O'Neill's original books, they made their work freely available on the internet (Chambers, 2011). Many of the tunes from O'Neill's books are played differently by musicians today, as is normal with a living tradition. Around the same period (the late 1990's) Henrik Norbeck collected nearly two thousand tunes in $\mathrm{ABC}$ notation from various sessions and recordings. Again this collection was made freely available on the internet. This collection contains many modern settings of tunes from O'Neill's books (Norbeck, 2011). Similarly, Bill Black a musician from Cape Cod, USA has transcribed a selection of Breathneach's books in $\mathrm{ABC}$ notation and made these freely available on the internet.

Web site thesession.org (thesession.org, 2011) contains an extensive; crowd-sourced collection of over eleven thousand traditional tunes in $\mathrm{ABC}$ notation entered by the traditional music community. The collection can be searched using text queries by any of the metadata associated with a tune or by melodic queries in $\mathrm{ABC}$ notation. The web

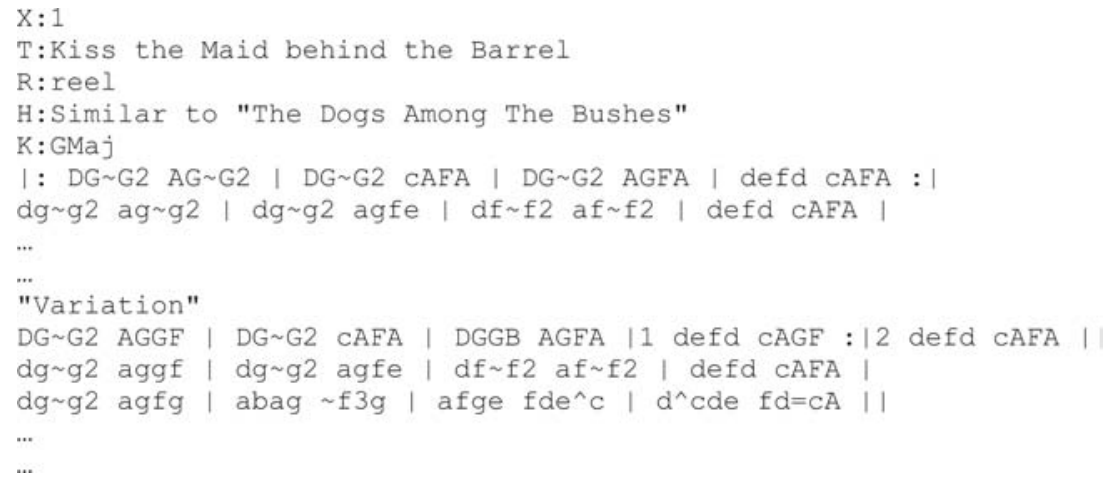

\section{Tunepal: searching a digital library}

287 
OCLC 27,4

288 site is significant, because it is supported by an active community of thousands of musicians who regularly contribute tunes, report on traditional music sessions and engage in lively discussions.

\section{The tunepal.org web site}

The tunepal.org web site (Figure 4) presents users with a search interface to a corpus of 16,700 tunes in $\mathrm{ABC}$ notation.

Users can enter a tune title or perform an advanced search by limiting matches to one or more tunebooks from the collection. To perform a query-by-playing (QBP) search (the most popular method of searching), a Java applet records a twelve second extract and transcribes the recording to $\mathrm{ABC}$ notation using an approach described in detail in (Duggan, 2009). The tunepal.org web site has been tested on a wide range of traditional instruments including the concert flute, tin-whistle, uilleann pipes, accordion, concertina and banjo - though it works best with instruments played "legato". The system compensates for various expressive elements in the user's playing such as the use of ornamentation (Duggan et al., 2009). Users are also offered the ability to change the transcription fundamental. This changes the frequencies used by the pitch spelling algorithm, so that tunepal.org can work with differently pitched instruments, such as Eb flutes and uilleann pipes pitched in B and C.

Matching is performed on the server using a variation of the classic edit distance algorithm (Navarro and Raffinot, 2002) against "search keys" - normalised strings of musical notes extracted from the scores during the import process (Duggan, 2009). These are stored in a MySQL database while matching is powered by a J2EE web application. QBP searches return the top ten matching tunes from the corpus in order of descending confidence. The web site tunepal.org gives the correct tune as the closest match for 93 percent of queries in experiments using field recordings of traditional musicians from sessions, classes, concerts and commercial recordings including solo and ensemble playing on traditional instruments recorded in a variety of real-world settings such as noisy public sessions (Duggan et al., 2009). The closest matching tune for each query is logged in the database each time a query is made. The web site incorporates a feedback system, so users can however proof listen to the results and give feedback as to which (if any) of the returned tunes was the correct one.

Each returned tune can be played back, displayed in $\mathrm{ABC}$ notation or staff notation. Additionally, each matching tune links to the original source of the $\mathrm{ABC}$ notation on the web, a discography for the tune (which in turn links to an Amazon MP3 store search) and a YouTube search for recordings of the tune can be made. Stave notation display uses ABCJS, an open source, browser hosted rendering engine for $\mathrm{ABC}$ notation (Dyke and Rosen, 2010) (Figure 5).

The Tunepal corpus aggregates collections of Welsh, Scottish, Breton, Canadian and American marching band music in addition to several different transcriptions of the same tune from the canon of Irish traditional music. Table I presents a breakdown of sources of the tunes in the Tunepal corpus.

The tunepal.org web site was launched on 31 July, 2009. It can be accessed on Windows, Mac and Linux systems running a variety of modern web browsers. The web site was promoted on popular traditional music discussion forums such as thesession.org and the Chiff and Fipple forum (Chiff \& Fipple, 2011). The web site has 


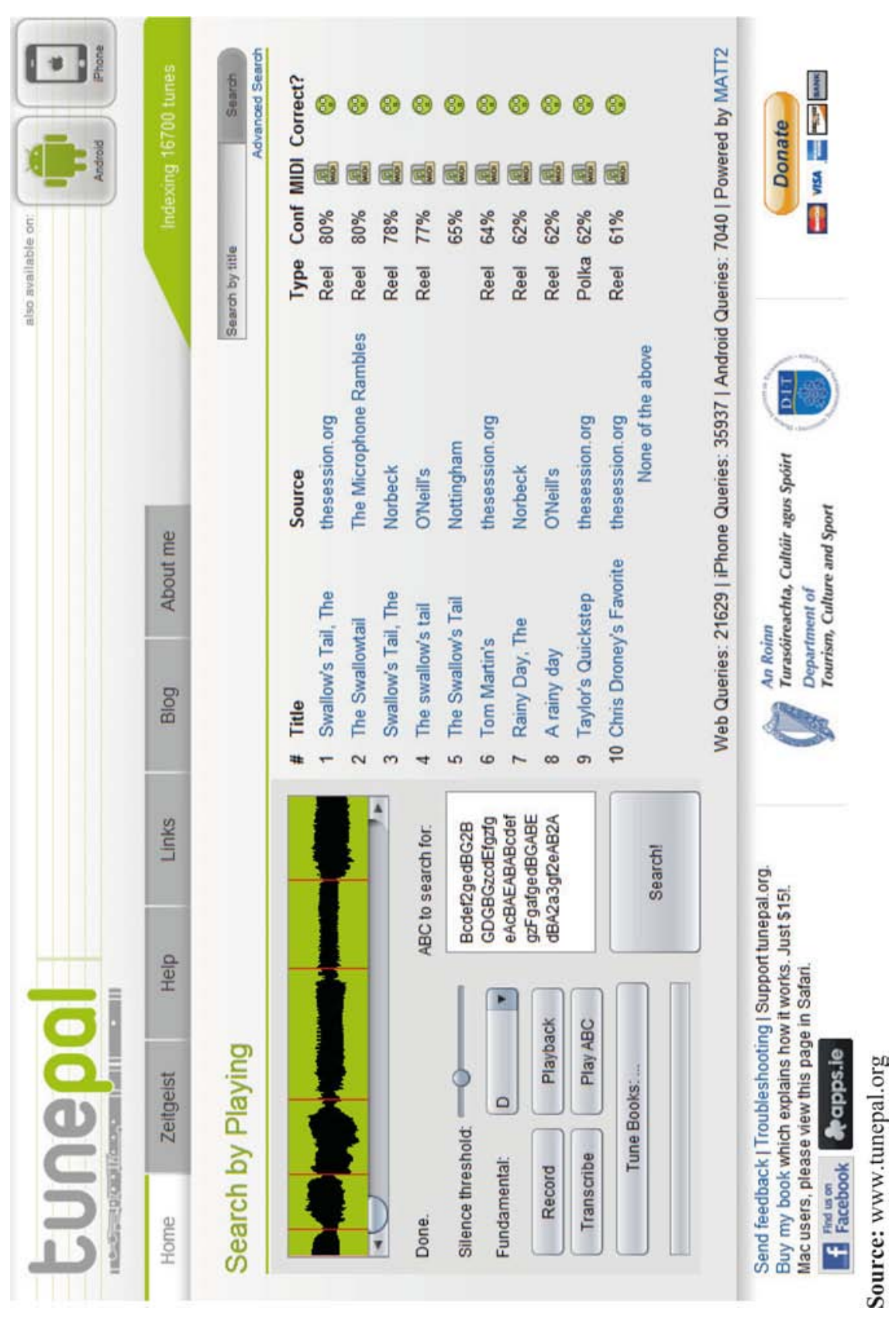

Tunepal: searching a digital library

289
Figure 4. Performing a QBP search with the tunepal.org web site 
OCLC

27,4

290

Figure 5.

Tune display from the web site tunepal.org

\section{Ciara And Dave's Wedding}

$\mathrm{X}: 8636$

I:Ciara And Dave's Wedding

$\mathrm{M}: 4 / 4$

L: $1 / 8$

R:hornpipe

$\mathrm{K}: \mathrm{Dmaj}$

(3DEF $|G B d B=c d e g| f d=c A . D E F D|G B d B=c d e g|$ fdef $\sim g 3 f \mid$

$\sim g 3 b$ afdf $\mid$ e=ceg $d B G F|G B d B=c d e g| f d=c$ AG2: |

I: fgladab $\sim$ g3dleggf $\sim d 3 g \mid a=c$ 'ba gbagled=ced2 (3efg |

$a d a b \sim g 3 b \mid=c$ 'bag e2de $\mid$ gged $g f=c e \mid d f=c e$ d2: |

Source: thesession.org

Stats:

2 tunepal.org QBP queries

0 Mobile QBP queries

40 mobile downloads

Download:

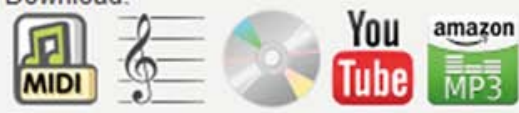

Share:

$35 \sqrt{6}+11$

Ciara And Dave's Wedding
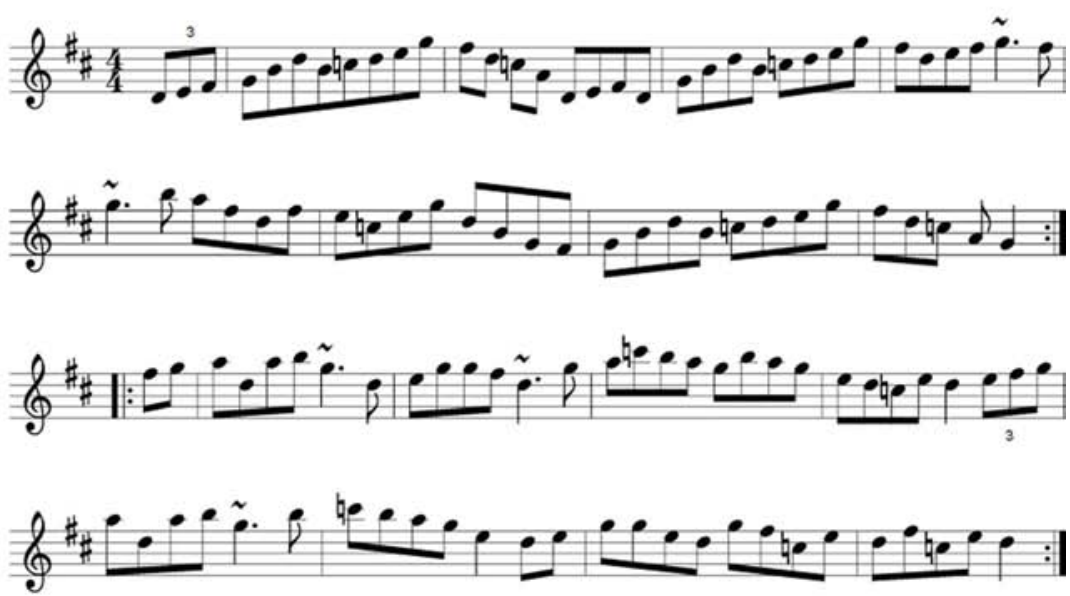

Using ABC]S by Greg Dyke and Paul Rosen

(2) 100

Source: www.tunepal.org 


\begin{tabular}{|c|c|c|}
\hline Source & Count & searching a \\
\hline thesession.org & 9,652 & digital library \\
\hline Henrik Norbeck & 1,474 & \\
\hline O’Neill's 1001 & 994 & \\
\hline Ceol Rince na hÉireann 1 & 73 & \\
\hline Ceol Rince na hÉireann 2 & 192 & 291 \\
\hline Ceol Rince na hÉireann 3 & 37 & \\
\hline Ceol Rince na hÉireann 4 & 220 & \\
\hline Johnny O'Leary & 196 & \\
\hline Nigel Gatherer & 794 & \\
\hline The Microphone Rambles & 194 & \\
\hline Welsh Music (John Tose) & 197 & \\
\hline Scottish Flute Music (Jack Campin) & 241 & \\
\hline Company of Fife and Drum & 238 & \\
\hline Nottingham Music Database & 1,034 & \\
\hline Aird's Airs (Jack Campin) & 1,164 & Table I. \\
\hline Total: & 16,700 & Sources of Tunepal tunes \\
\hline
\end{tabular}

proved very successful and is now well known amongst traditional musicians having been profiled in several Irish national newspapers (Long, 2009; Lillington, 2010).

\section{Tunepal mobile apps}

Traditional Irish music is most commonly played by groups of musicians in a community setting known as a session (O'Shea, 2006). Sessions usually take place in shared public spaces in the evening times and often take place in pubs. The Tunepal mobile apps can be conveniently and discretely used in traditional music sessions to access to the collections described in section 4 . There are optimised, native mobile clients for iPhone/iPod Touch (Figure 6), Google's Android OS and a special version known as Tunepal HD for iPad. All the apps offer identical functionality, but Tunepal $\mathrm{HD}$ has a redesigned user interface which takes advantage of the bigger screen size on the iPad. In common with the tunepal.org web site, the apps support queries by tune title or by playing on a traditional instrument. The Tunepal apps allow searches over the entire tunepal.org tune corpus. This is not in fact stored on the devices themselves. Instead, the corpus is stored "in the cloud" on the tunepal.org server and tunes are downloaded on demand. For query-by-playing (QBP) searches, 12 seconds of audio is sampled at 22Khz, transcribed as described in (Duggan, 2010) and submitted to the tunepal.org J2EE web service for matching. When a tune is retrieved using Tunepal, it can be played back, transposed, speeded up or slowed down, displayed in ABC or stave notation, emailed or posted to Facebook. Additionally a discography can be retrieved, which refers the user to iTunes or the Amazon MP3 store to purchase the recording. YouTube search results for a tune can also be displayed. The Tunepal apps also allow users to edit music scores in $\mathrm{ABC}$ notation and to compose new tunes that can be instantly displayed in stave notation or played back. Figure 6 illustrates a typical workflow - recording a query, finding a match, displaying the stave, retrieving the discography, performing a YouTube search for a recording of the tune and finally, posting the tune to Facebook. 


\section{OCLC}

27,4

292

Figure 6.

Screenshots of Tunepal running on an iPod Touch illustrating a typical workflow

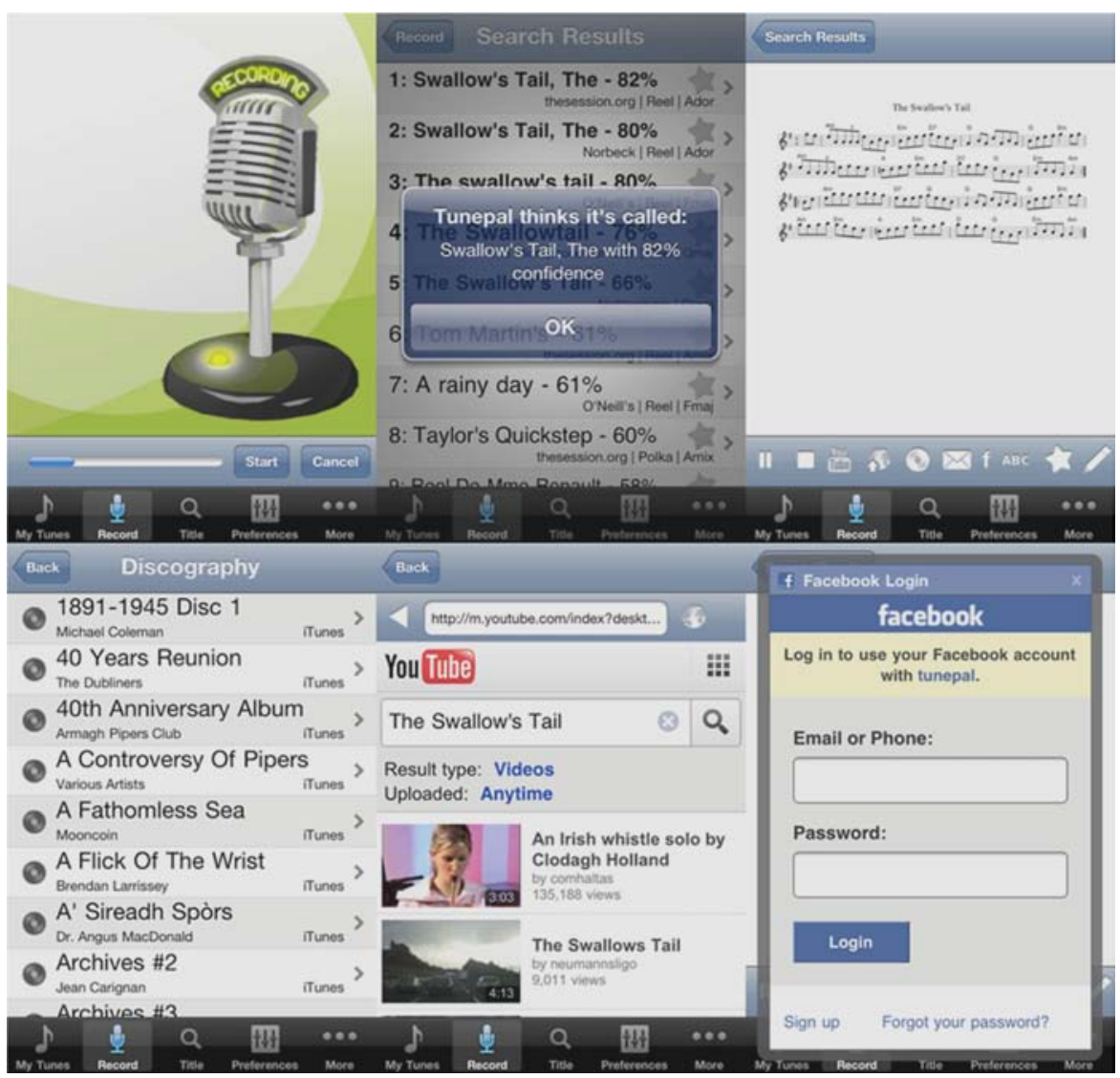

Retrieved tunes are stored in a "My Tunes" section on the user's device, in order of most recently found to facilitate future retrieval for learning purposes. Playback is achieved using ABC2MIDI (Shlien, 2011) and the FMOD audio engine on iOS devices (FMOD, 2011). Android devices support MIDI playback natively. The Tunepal mobile apps also allow the user to switch the interface between Irish Gaelic (Figure 7) and English. The mobile versions of Tunepal have one major advantage over the tunepal.org web site and that is the ability for accurate geocoding of queries.

With the users permission, each query made using the mobile apps is geotagged and the latitude and longitude is logged in the tunepal.org database. This makes it possible for users to track their queries on a map (Figure 7) on their devices.

Tunepal for iPhone was released on 11 February, 2010. This was followed on 1 July 2010 with Tunepal for Android and on 15 December 2010 with Tunepal HD for iPad. New versions of the apps with additional functionality and accuracy were released at intervals culminating in a public press launch for all the apps on 15 December 2010 attended by luminaries from the world of traditional music. The apps are now in use by thousands of users in 29 countries worldwide. 


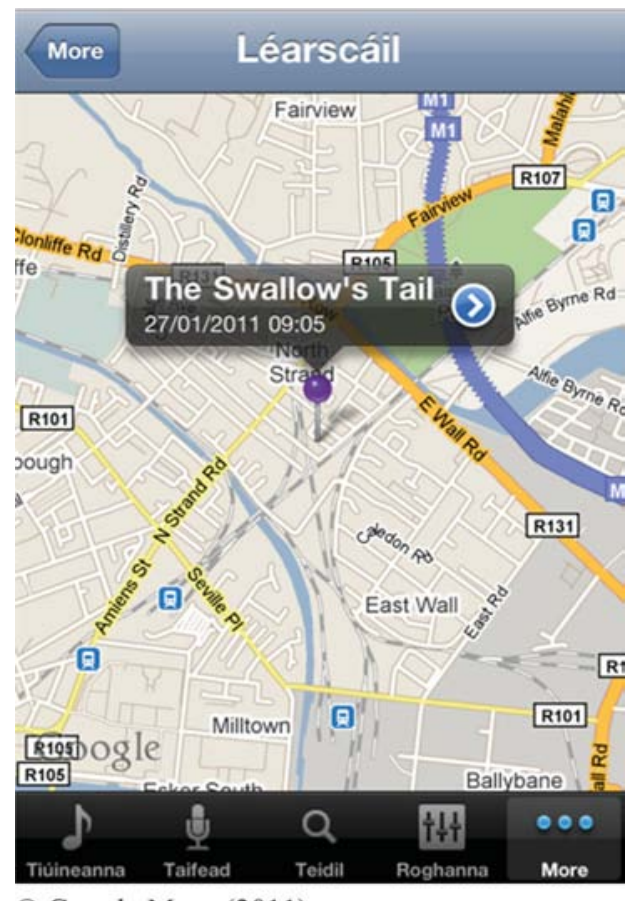

\section{Tunepal: searching a digital library}

293

C Google Maps (2011)

\section{Dissemination}

At the time of writing (26 January 2011), 53,403 queries have been logged by iOS users, while 10,095 queries have been logged by Android OS users. A total 20,813 QBP queries have been logged through the web site. This represents a total of 84,311 tune queries. Table II presents a breakdown of mobile queries by OS.

A total of 74 percent of queries made on mobile devices are geotagged. Google maps plotting the usage of Tunepal geographically were developed so that the geographic profile of Tunepal usage could be visualised. The maps display a drop pin for each geotagged query on the map. Pins link to callouts that display the closest matching tune for each query and an icon indicating whether the user was on an iOS or Android OS device. Live maps plotting the last two thousand geotagged queries performed can be viewed at the web site tunepal.org. Figure 8 shows a selection of geotagged QBP queries on the map of Ireland.

In order to get a numeric breakdown of where these queries were being made, 30,167 geotagged QBP queries were reverse geocoded using the Google Maps web service to

\begin{tabular}{lrcccrr}
\hline & iOS QBP & Android OS QBP & iOS Title & Android OS Title & Total & \\
Geotagged & 25,032 & 5,135 & 13,755 & 3,118 & 47,040 & \\
Non-Geotagged & 8,704 & 1,096 & 5,912 & 746 & 16,458 & A breakdown of Tunepal \\
Total & 33,736 & 6,231 & 19,667 & 3,864 & 63,498 & mobile queries \\
\hline
\end{tabular}




\section{OCLC}

27,4

\section{4}

\section{Figure 8.}

Geotagged Tunepal mobile QBP queries plotted on a Google map (see tunepal.org)

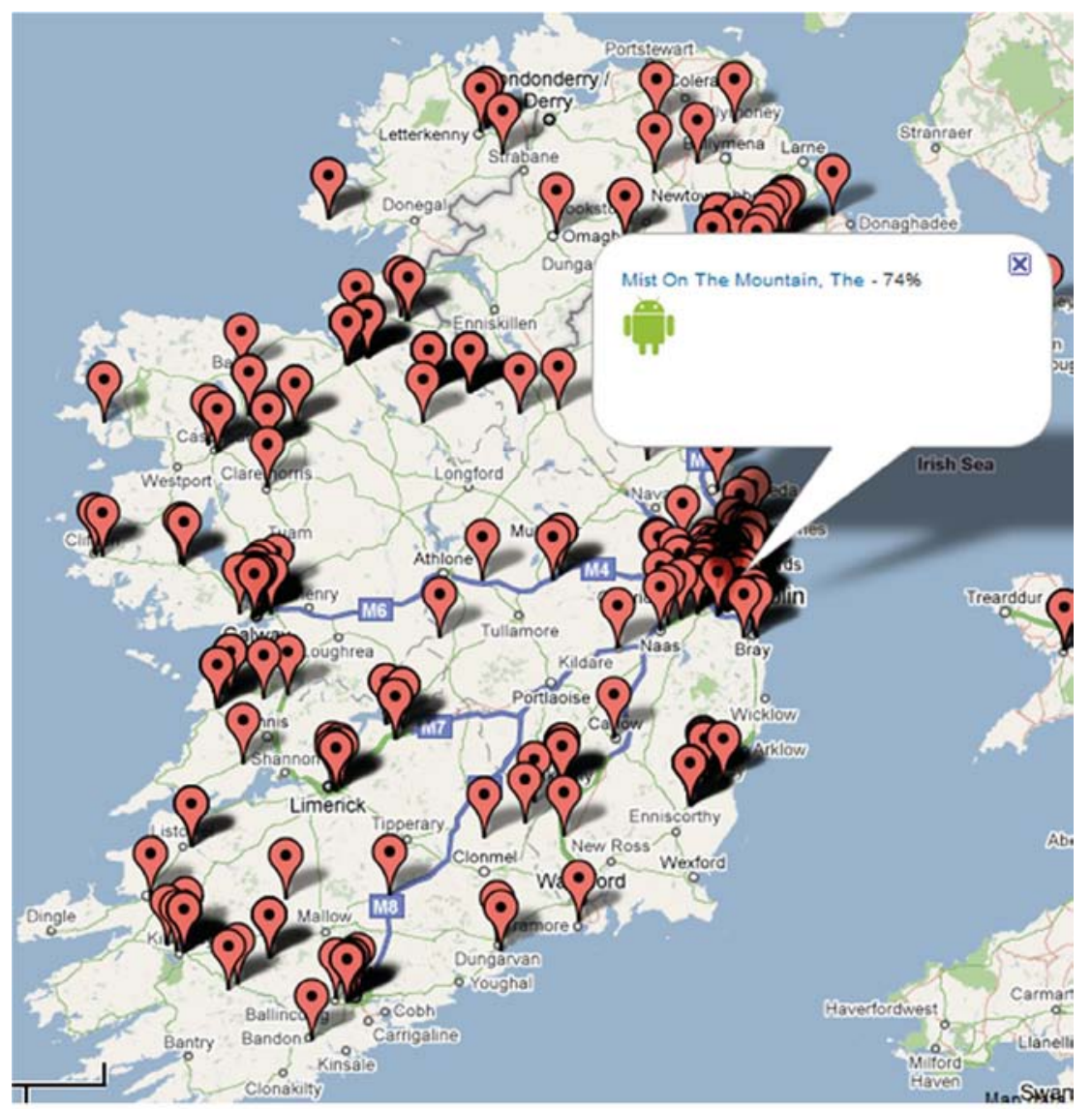

(C) Google Maps (2011)

Note: See www.tunepal.org

retrieve the approximate geographic address. This revealed that queries originated from 29 different countries. Table III shows the top 20 countries in descending order.

It is significant that the majority of international queries originate in the USA and the UK, these being major centers for the Irish diaspora and also countries where traditional music sessions take place regularly. Interestingly Japan is also a significant source of queries, reflecting the growing popularity of traditional Irish music and culture in that country.

Analysis of the queries of Irish origin (of which there are 10,613) reveals the top counties in Ireland for queries. Dublin, being the capital generates significantly more queries than the other counties; however Galway and Clare are also significant sources of queries, these being centres of Irish music and culture. In particular county Clare hosts a number of prominent traditional music festivals, where aficionados of traditional music from around the world gather to play and listen to Irish music. By plotting the Irish queries over the course of the day, a profile can be established as to 


\begin{tabular}{lr}
\hline Country & Query count \\
\hline Ireland & 10,613 \\
USA & 9,093 \\
UK & 4,507 \\
Canada & 1,136 \\
Australia & 1,046 \\
France & 987 \\
Germany & 804 \\
Japan & 695 \\
Denmark & 209 \\
Netherlands & 195 \\
Spain & 165 \\
Sweden & 148 \\
Italia & 126 \\
New Zealand & 98 \\
Croatia & 66 \\
Switzerland & 58 \\
Romania & 43 \\
Norway & 41 \\
India & 39 \\
South Africa & 23 \\
\hline
\end{tabular}

\section{Tunepal: searching a digital library \\ 295}

when traditional music is most commonly played. The x-axis in Figure 9 is the hour of the day in 24-hour format, while the number of queries is on the y-axis.

The hypothesis that the Tunepal mobile apps are most commonly used in traditional music sessions is clearly supported by this profile. Traditional music sessions most commonly take place in the late evenings and this is when the majority of queries are made.

Tunepal for iPhone was listed in the top twenty cultural apps available on the iPhone by the Sunday Times (an Irish national newspaper) alongside apps such as Shazam and Spotify, IMDB and an app from the British National Gallery (Sunday Times, 2010). Tunepal has also been extensively discussed on traditional Irish music forums (Chiff \& Fipple Forum, 2010).

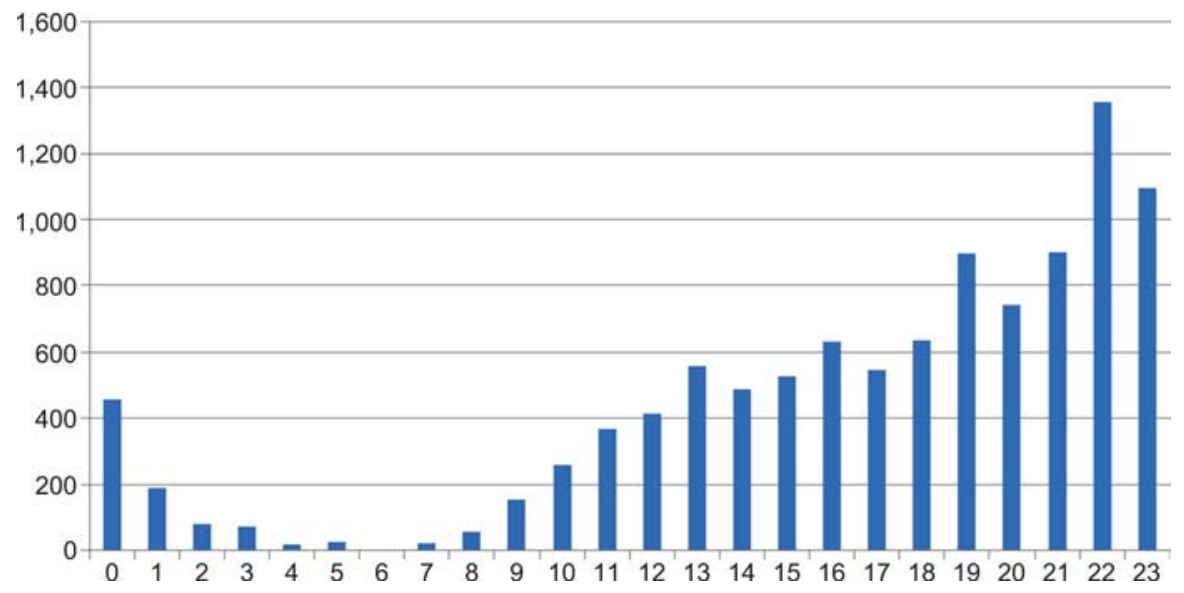

Figure 9. QBP queries originating in Ireland plotted by time of day 
OCLC

27,4

296

\section{8, Conclusions and future work}

In this paper Tunepal was presented - a query-by-playing digital library project for traditional music that developed from academic research. The Tunepal tools have become very popular, being used by musicians around the world to connect their playing with scores, tune names and biographic data. To achieve this, a corpus of 16,700 compositions collected by both the traditional music community and noted collectors such as O'Neill and Breathnach has been aggregated in the Tunepal database. Interfaces have been developed to allow this database to be searched by title or by playing an audio query on a traditional instrument either over the web or on mobile devices such as the iPhone, iPad or Android OS Phones. Matching tunes can be downloaded to a mobile device, edited, displayed in ABC or stave notation, played back and transposed. Tunepal also facilitates discography, YouTube, iTunes and Amazon music searches for tunes. This paper also presented an analysis of the logs collected on the usage of these systems since launch.

Tunepal has been favorably received and musicians report that the system is being used in variety of scenarios including archiving and the preparation of sleeve notes for commercial recordings. Nevertheless, a minority of musicians report skepticism of the use of technology in traditional music sessions (ironically on internet forums).

In future work it is hoped to expand the corpus of tunes to include many more tunes from different traditions. Additionally it is hoped to be able to link Tunepal with digital archives of recordings and videos of traditional music as it is felt that this will greatly enhance the potential for Tunepal to fulfill its role in the learning and dissemination of traditional music worldwide.

\section{References}

Breathnach, B. (1999), Ceol Rince na hÉireann Cuid V (Dance Music of Ireland), Vol. V, An Gúm, Baile Átha Cliath.

Bunting, E. (1840), The Ancient Music of Ireland: Arranged for Piano, Hodges and Smith, Dublin. Chambers, J. (2011), O’Neills Books, available at: http://trillian.mit.edu/ jc/music/book/oneills/.

Chiff \& Fipple Forum (2010), "View topic - after forty years playing this music ...", Chiff \& Fipple Forums, available at: http://forums.chiffandfipple.com/viewtopic. $\mathrm{php} ? \mathrm{f}=2 \& \mathrm{t}=75943$ (accessed June 16, 2010).

Chiff \& Fipple (2011), Chiff \& Fipple Forums, available at: http://chiffboard.mati.ca/.

Driscoll, S. (2004), “A trio of internet stars: ABC's”, Fiddler Magazine, Vol. 11 No. 2, Los Altos, CA, June 1.

Duggan, B. (2009), "Machine annotation of traditional Irish dance music", PhD, School of Computing, Dublin Institute of Technology, Dublin.

Duggan, B. (2010), "Tunepal: the traditional musician's toolbox", in Proceedings of the Second Workshop on EHeritage and Digital Art Preservation, ACM, 25-29 October, Firenze, Italy.

Duggan, B., O'Shea, B., Gainza, M. and Cunningham, P. (2009), "Compensating for expressiveness in queries to a content based music information retrieval system", 2009 International Computer Music Conference, Montreal, Aug 16-21.

Dyke, G. and Rosen, P. (2010), "abcjs - Project Hosting on Google Code", available at: http://code. google.com/p/abcjs/ (accessed March 21, 2010).

FMOD (2011), "fmod - interactive audio middleware", FMOD, Melbourne, available at: www. fmod.org/ (accessed March 21, 2010).

Joyce, W. (1909), Old Irish Folk Music and Song, Longmans, Green, London. 
Larsen, G. (2003), The Essential Guide to Irish Flute and Tin Whistle, Mel Bay Publications, Inc, Pacific, MO.

Lillington, K. (2010), "Technology to track trad”, The Irish Times, available at: www.irishtimes. com/newspaper/finance/2010/0716/1224274815780.html (accessed August 17, 2010).

Long, S. (2009), "Film body makes case against bord snips", The Irish Times, Saturday 8 August.

Mansfield, S. (2011), "How to interpret ABC notation", available at: www.lesession.co.uk/abc/ abc_notation.htm

Navarro, G. and Raffinot, M. (2002), Flexible Pattern Matching in Strings: Practical On-Line Search Algorithms for Texts and Biological Sequences, Cambridge University Press, Cambridge.

Norbeck, H. (2011), “ABC tunes”, available at: www.norbeck.nu/abc/index.html (accessed January 27, 2011).

O'Shea, H. (2006), "Getting to the heart of the music: idealizing musical community and Irish traditional music sessions", Journal of the Society for Musicology in Ireland, Vol. 2 No. 7, p. 1.

Petrie, G. (1855), The Petrie Collection of the Ancient Music of Ireland.

Shields, H. (1998), Tunes of the Munster Pipers: Irish Traditional Music from the James Goodman Manuscripts, Irish Traditional Music Archive, Dublin.

Shlien, S. (2011), "The ABC music project - abcMIDI", available at: http://abc.sourceforge.net/ abcMIDI/ (accessed January 27, 2011).

(The) Sunday Times (2010), "The arts world on iPhone", The Sunday Times, March 28.

thesession.org (2011), "The session.org Forums", available at: www.thesession.org.

Vallely, F. (1999), The Companion to Irish Traditional Music, New York University Press, New York, NY.

Wallis, G. and Wilson, S. (2001), The Rough Guide to Irish Music, Rough Guides, London.

Walshaw, C. (2011), "The ABC home page”, available at: www.walshaw.plus.com/abc/

\begin{abstract}
About the authors
Bryan Duggan is a Lecturer in the School of Computing in the Dublin Institute of Technology. He received his $\mathrm{PhD}$ on the topic of "Machine annotation of traditional Irish dance tunes" in October 2009. This work proposed new algorithms and systems for the analysis of recordings of traditional Irish dance music. He has published extensively on the topic of query-by-playing music information retrieval systems. Bryan is also the author of Tunepal for iOS and Android, popular apps used by thousands of musicians around the world to search for music. In his spare time, Bryan enjoys playing traditional Irish music on the concert flute. Bryan Duggan is the corresponding author and can be contacted at: bryan.duggan@dit.ie

Professor Brendan O'Shea graduated from NUI Galway with a BSc and MSc in Mathematics. He pursued his $\mathrm{PhD}$ studies and research at Royal Holloway College (London University) and University of Nebraska. His topic of research was "Efficiency of algorithm for solving large sets of coupled differential equations". Having graduated with PhD from London University in 1971 he took up an appointment as Lecturer in the Department of Mathematics and Computer Science at Dublin Institute of Technology. He progressed to Senior Lecturer, Assistant Head of Department and most recently Professor and Head, School of Computing.
\end{abstract}

To purchase reprints of this article please e-mail: reprints@emeraldinsight.com Or visit our web site for further details: www.emeraldinsight.com/reprints 